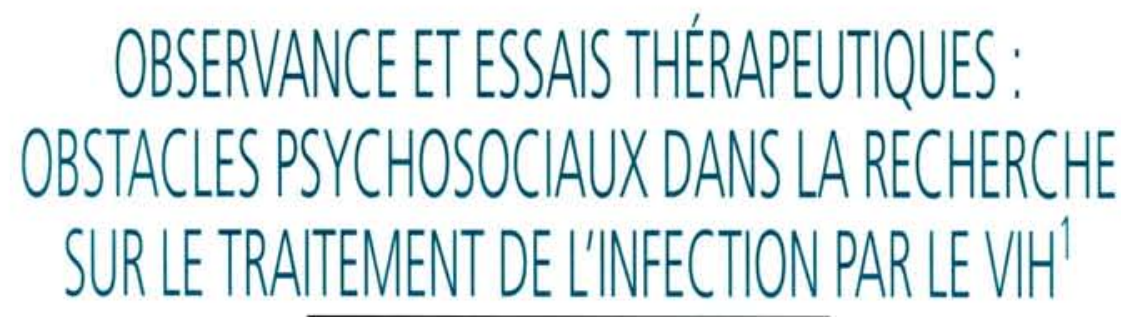

MICHEL MORIN ET JEAN-PAUL MOATII

Les débuts de l'année 1996 ont remis au premier plan de l'actualité l'importance des attentes et des impatiences concernant les innovations thérapeutiques que la recherche sur le sida permet dès maintenant d'entrevoir. Après une longue série de déceptions et d'imprudences médiatiques, les chercheurs ont pu présenter consensuellement et à partir de résultats d'essais cliniques, des recommandations renouvelées concernant les stratégies de soins pour les personnes atteintes par le VIH (Yéni, 1996). On sait maintenant que s'il n'existe toujours pas de traitement qui conduise à la guérison, les progrès thérapeutiques, en particulier en matière d'infections opportunistes, permettent d'allonger la survie des personnes infectées par le VIH. Après les premiers résultats positifs obtenus avec l'AZT dans les traitements antiviraux proprement dits, le nombre des molécules antivirales candidates susceptibles d'être expérimentées continue à s'élargir et, depuis la $\mathrm{III}^{\mathrm{e}}$ conférence internationale de Washington (janvier 1996) sur les rétrovirus et les infections opportunistes, les traitements associant plusieurs antiviraux entre eux (AZT, ddI, ddC, 3TC, antiprotéase, etc.) tendent à devenir le standard thérapeutique. On parle, avec une assurance grandissante, d'une "chronicisation" contrôlée de la pathologie associée à l'infection.

Cette nouvelle orientation du soin s'accompagne d'une transformation importante dans les représentations scientifiques de l'histoire naturelle de la maladie. Les avancées de la recherche ont en effet mis en cause la distinction entre la phase asymptomatique* de l'infection à VIH et la maladie sida proprement dite, distinction qui a long. temps guidé les attitudes thérapeutiques et organisé les représentations collectives de la lutte contre l'épidémie. La récente mise en

\section{RÉSUMÉ : Observance et essais thérapeutiques : obstacles psychosociaux dans la recherche sur le traitement de l'infection par le VIH}

L'existence de comportements de non-observance des prescriptions thérapeutiques ou préventives par les patients a été documentée depuis longtemps par la sociologie médicale au point que certains $y$ voient le problème le plus sérieux auquel la pratique médicale moderne a à faire face. L'épidémie d'infection à VIH a joué un rôle de révélateur social de ce phénomène, qui semble notamment exacerbé dans le cadre des essais cliniques pourtant indispensables à la définition de stratégies thérapeutiques efficaces face au sida. L'article fait état d'une recherche qualitative menée auprès de soignants et de patients d'un centre hospitalier du Sud-Est de la France concernés par l'un des principaux essais cliniques récents (Essai Delta) dans le domaine du sida. Ce travail est l'occasion de discuter les limites des approches psychosociales d'inspiration nord-américaine utilisées jusqu'à présent dans l'étude de la non-observance et d'ébaucher une réflexion alternative fondée sur la théorie des représentations sociales. évidence dans la presse scientifique internationale (Ho et al., 1995) que cette latence apparente masque en réalité une intense activité du VIH va désormais venir justifier des interventions thérapeutiques les plus précoces possibles, voire même dès la primoinfection, c'est-à-dire dans les deux à trois semaines suivant la contamination initiale.

Dans le même temps, cependant, en dépit ou à cause de ces progrès et de ce retour tant attendu de signes de succès des sciences médicales, cliniciens et chercheurs doivent faire face à des difficultés qu'on peut qualifier de "psychosociales". Ces difficultés, pour n'être pas nouvelles, ont pris récemment un relief particulier. La France a ainsi été secouée pendant quelques semaines d'un débat vertueux quand on a médiatiquement découvert que des médicaments (les antiprotéases), nouvellement crédités aux États-Unis d'effets positifs démontrables, semblaient pouvoir être attribués à tous les patients susceptibles d'en bénéficier. On s'est particulièrement indigné de la prise de position du Conseil National du sida qui, en cas de pénurie effective de ces molécules, envisageait un tirage au sort des malades qui pourraient recevoir le nouveau médicament dans le cadre d'expérimentations thérapeutiques. Cet événement, dramatiquement monté en épingle, n'était que le plus récent d'une histoire tumultueuse qui expose les patients à des informations conflictuelles ou contradictoires dans le débat public permanent qui accompagne le sida depuis sa découverte (Herzlich et Pierret, 1988 ; Grmek, 1989). Le décalage entre chercheurs 
et grand public reste en fait considérable et l'incertitude est encore très prégnante parmi les patients, ballottés entre les recommandations médicales et les mises en garde des associations qui tentent de défendre leurs intérêts.

C'est dans ce contexte d'espoir et de perplexité que l'on voit grandir depuis quelques temps des préoccupations qui touchent une condition essentielle des avancées de la recherche de voies thérapeutiques efficaces : l'organisation et le devenir des essais cliniques $^{*}$. La multiplication de ces essais met en jeu des intérêts multiples et devient une épreuve de vérité essentielle pour un nombre grandissant d'acteurs engagés dans la lutte contre l'épidémie :

- pour de nombreuses équipes de recherche qui sont impliquées dans une course d'urgence concurrentielle aux résultats validés ;

- pour les cliniciens et les soignants qui sont de plus en plus amenés à intégrer une activité de collaboration aux essais ou à la recherche dans leur pratique quotidienne ;

- pour les patients qui, à l'occasion d'une sollicitation de participer à un essai, peuvent être entraînés dans une épreuve de vérité personnelle mettant profondément en cause leur rapport à la maladie, à leur entourage et à la relation médecin-malade.

Ces questions commencent seulement à accéder à un seuil de visibilité notable et leur ampleur est généralement minorée. On est amené pourtant de plus en plus à s'inquiéter du recrutement nécessaire de volontaires disponibles en nombre suffisant pour des protocoles dont la durée est souvent longue malgré la créativité développée par les chercheurs pour la raccourcir. On tente de prévoir et d'expliquer des abandons ou disparitions pour raisons extra-médicales qui paraissent anormalement importants pour certains essais. Plus qualitativement, on observe que les comportements et les attitudes de nombreux patients s'écartent parfois de manière importante, et souvent à l'insu des médecins, de ce qui est prescrit, mettant ainsi en péril l'interprétation des résultats. Il devient donc urgent de faire le point sur ces problèmes qui sont encore trop souvent cantonnés dans une polémique dramatisée ou une dénégation ironique. C'est, selon nous, aux sciences sociales de travailler maintenant à éclairer des enjeux et des processus dont l'apparente nouveauté spectaculaire masque le caractère fondamental pour le développement des relations de soin qui s'articulent sur la recherche médicale. Nous illustrerons ce point de vue en présentant un bilan partiel des travaux encore rares ou méconnus qui se sont attaqués aux mystères de ce que la psychologie médicale traite sous les termes génériques de compliance et d'adhérence, ou dans la dénomination de langue française que nous avons retenue, d'"observance thérapeutique" qui fait référence au " respect des prescriptions médicales par les patients " (Garnier et al., 1992). C'est dans ce cadre que nous avons voulu interroger certains aspects critiques de la recherche clinique sur l'infection par le VIH.
* Les mots suivis d'un astérisque sont définis dans le glossaire.

1. Recherche menée grâce au soutien de I'Agence Nationale de Recherche sur le Sida (66bis, avenue Jean Moulin, 75014 Paris) et à la coopération active du Professeur Jean-Albert Gastaut (Hôpital de Jour, Hôpital Sainte Marguerite, Marseille).

La recherche dont on présente ici le cadre théorique et quelques illustrations se traduit dans plusieurs opérations spécifiques de terrain actuellement en cours qui sont placées sous la responsabilité de Marc Souville et Nathalie

Munzenberger, psychosociologues attachés à l'Unitè INSERM 379. 


\section{GLOSSAIRE}

Asymptomatique : c'est l'absence de symptôme clinique.

Essai clinique : il s'agit de l'étude de la première administration, de l'efficacité pharmacologique et de l'efficacité thérapeutique d'une substance chimique. Cette étude des médicaments, chez I'homme, est strictement définie en France dans le cadre de la loi Huriet du 20 décembre 1988.

Les essais sur I'homme se répartissent en quatre phases, mais nos recherches s'intéressent plus particulièrement aux essais de phase II et III :

- Essais de phase I: étude de la première administration chez l'homme; détermination de la posologie entraînant les premiers effets indésirables et de la posologie entraînant les premiers effets pharmacodynamiques souhaités.

- Essais de phase II : étude de l'efficacité pharmacologique d'une substance chimique : détermination de la dose optimale et précision des effets indésirables et de leurs symptômes.

- Essais de phase III : essais comparatifs ; évaluation de l'efficacité thérapeutique : conditions optimales de prescriptions ; tolérance du produit testé. Ces essais par leur nature durent longtemps (généralement de un an à quatre ans) et portent sur des populations importantes pouvant aller jusqu'à plusieurs milliers de patients (Bouvenot et Vray, 1994).
- Essais de phase IV : après la mise sur le marché ; études d'efficacité et de tolérance dans les conditions usuelles de prescription. On cherche à déceler des effets secondaires rares. Cette phase débouche sur la pharmacovigilance.

Essai en double-aveugle : signifie que ni le patient, ni les soignants (médecins, infirmières, etc.) qui le prennent en charge ne savent quel est le traitement administré.

Placebo : substance dépourvue d'activité pharmacodynamique (qui est neutre) mais dont I'administration peut produire un effet thérapeutique (appelé "effet placebo"), le malade la considérant comme un médicament.

Randomisation : procédure qui consiste à répartir au hasard, par tirage au sort, les sujets recrutés pour l'essai dans chacun des groupes constitués pour cet essai. C'est la seule manière de garantir qu'une différence statistiquement significative de résultats thérapeutiques entre deux groupes est bien attribuable à la différence des traitements administrés.

T4 (ou CD4+) : variété de globules blancs, participant à l'immunité de l'organisme. Le "taux de T4" est le nombre de T4 que l'on compte dans un $\mathrm{mm}^{3}$ de sang. Il est normalement compris entre 500 et $1200 / \mathrm{mm}^{3}$, mais il peut diminuer dans certaines situations, notamment dans le cas d'une infection par le $\mathrm{VIH}$.

\section{LES CONSTATS DE NON-OBSERVANCE CHEZ LES PATIENTS INFECTÉS PAR LE VIH}

\section{De l'insoumission en prévention primaire...}

La mission la moins discutée pour les sciences sociales dans la lutte contre le sida a été de collaborer à la prévention primaire des risques d'infection par le VIH. On leur a demandé de faciliter, au niveau du grand public, l'adoption de comportements permettant d'éviter la transmission du virus par la voie sexuelle ou intraveineuse, en aidant aux campagnes en faveur du préservatif par exemple, ou à celles qui préconisent un recours volontaire aux tests de dépis- tage lorsqu'un risque d'infection est soupçonné. Des données se sont accumulées qui montrent que connaître et reconnaître l'intérêt d'une mesure préventive ne garantit en rien l'adoption et l'appropriation individuelles durables de cette mesure. On ne se soumet à la prévention qu'à certaines conditions que tentent d'identifier et contrôler de manière prédictive les sciences sociales. Par exemple, des travaux expérimentaux ont montré que le recours au dépistage du VIH sera facilité si l'on y est amené par une stratégie d'engagement volontaire associée à une discussion de groupe qui débat ouvertement de la pertinence des préconisations médicales (Morin et Joule, 1991 ; Joule et Peyrolle, à paraître). Sans renoncer aux apports démontrés des stratégies communicationnelles persuasives illustrées par les campagnes média- tiques d'information (Moatti et al., 1992), on s'attache à mieux comprendre pourquoi et surtout comment se construisent différentes "logiques préventives" face au risque (Paicheler et Quemin, 1994), ces logiques décalées s'écartant notablement des connaissances assimilées et des modèles de comportements prescrits, notamment pour ceux que l'on appelle "les jeunes" (Peto et al., 1992 ; Morin, 1994).

Ainsi, des études nord-américaines ont mis en évidence des délais importants dans la réalisation effective des tests de dépistage prescrits par le médecin et dans le recours ultérieur au système de santé après notification du diagnostic initial de séropositivité. Il en est de même pour le recours médical après apparition des premiers symptômes cliniques. Une étude nord-américaine établissait, par exemple, un délai médian de 104 jours entre la remise du diagnostic de séropositivité au VIH et la première visite médicale, $25 \%$ des patients attendant un an ou plus. Les raisons invoquées pour ce "retard" dans la recherche d'une prise en charge médicale étaient l'absence de couverture assurantielle, la méconnaissance de la nécessité d'un suivi médical, et la "peur" de se confronter au médecin. La longueur du délai était significativement associée au fait de ne pas avoir d'emploi, de ne pas bénéficier d'un soutien de l'entourage et de recourir au "déni" de la maladie ou à l'"espérance d'un miracle" pour gérer psychologiquement la situation (Katz et al., 1992).

\section{... aux mises en péril des essais cliniques}

Les évaluations menées après des essais de l'AZT sur des patients "asymptomatiques" ont révélé des taux élevés d'abandons et de perdus de vue (de l'ordre de $30 \%$ ) qui ont suscité des inquiétudes des responsables américains, notamment au niveau du NIH (National Institutes of Health). Quand des investigations psychosociales approfondies ont été menées auprès de 240 patients de San Francisco originel. lement inclus dans l'essai P019² (AZT ver. sus placebo chez des patients asymptoma- 
tiques avec $\mathrm{T} 4^{*}<500 / \mathrm{mm}^{3}$ ), dont près de la moitié n'ont pas terminé l'essai, on a constaté que la grande majorité des abandons n'était pas directement liée aux effets toxiques et secondaires de la molécule, mais plutôt à des motivations et choix des sujets, fortement corrélés à une insertion dans des groupes apportant un soutien communautaire (chez les homosexuels en particulier) et à un niveau d'information élevé (Chesney et Folkman, 1994). De plus, près d'un quart des sujets interviewés, demeurant dans l'essai, ont reconnu ne pas avoir respecté les consignes de l'essai en recourant à des prises indépendantes d'AZT. Des données concordantes suggèrent donc l'élaboration par certains patients, du moins dans le contexte nord-américain, de stratégies individuelles enracinées dans des normes d'appartenance à des groupes ; ces stratégies forment la base de jeux complexes encadrés par les objectifs collectifs de recherche des essais cliniques. Ainsi on a pu observer que certains patients avaient appris à utiliser tactiquement une information médicale comme l'utilisation du volume globulaire moyen, dont l'élévation est reliée à la prise d'AZT. Ils devenaient ainsi capables d'identifier le bras placebo* d'un essai censé être en double aveugle* (Becker et al., 1993).

Il est difficile, en l'état actuel, de dire si l'on rencontre une fragilisation équivalente des essais cliniques en France. Le débat en effet y a surtout été focalisé jusqu'à maintenant sur la difficulté et l'importance d'un équilibrage entre des contraintes de méthodologie scientifique indispensables aux protocoles, et des exigences éthiques et culturelles fondamentales pour la recherche médicale dans les pays démocratiques (Dormont, 1992 ; Lazar, 1996). Quelques études encore rares montrent l'importance des processus psychosociaux et notamment l'emprise des phénomènes relationnels qui interviennent dans la régulation ordinaire des essais cliniques (Fauché et al., 1994). Tout récemment, les premières évaluations de l'essai franco-britannique Delta (voir encadré) ont bien montré que les réactions imprévues ou non contrôlées des patients aux essais ne sont pas une spécificité nord-américaine. Cet essai a eu une grande importance par le nombre de sujets impliqués (2 191 pour l'essai Delta 1 et 1117 pour l'essai Delta 2) et par ses enjeux cliniques puisqu'il conduisait à préconiser des thérapeutiques combinant au moins deux molécules antivirales plutôt qu'une monothérapie par l'AZT. On a relevé cependant, dans une des dernières phases d'analyse de cet essai, que $65 \%$ des patients inclus avaient arrêté de suivre le traitement initial de randomisa. tion. Parmi eux "seulement" $13 \%$ des arrêts étaient expliqués par des manifestations cliniques ou par le décès. Parmi ceux dont on avait dû modifier le traitement, conduisant à une modification du protocole initial de l'essai, il s'agissait dans $15 \%$ des cas d'une baisse des T4, pour $22 \%$ d'effets secondaires et pour $39 \%$ du motif flou de "demande du patient" (Yéni, 1995 ; Chambon, 1995).

\section{USURE ET EMBARRAS DES MODĖLES EXPLICATIFS DE LA NON-OBSERVANCE}

\section{Pourquoi les toxicomanes sont-ils moins observants ?}

Les études nord-américaines évoquées plus haut constatent toute une moindre observance thérapeutique chez les patients toxicomanes en comparaison d'autres populations. Mais, quand elles approfondissent leurs investigations, elles constatent également un moindre accès aux soins pour les toxicomanes et quand celui-ci a lieu, c'est le suivi thérapeutique de ces patients qui est entravé. Par exemple, dans l'étude de Stein et al. (1991), à stade et à niveau de T4 équivalents, les toxicomanes séropositifs avaient une probabilité plus de deux fois inférieure aux autres patients d'avoir bénéficié de prescriptions d'AZT. Cet exemple illustre le risque du biais de "prophétie auto-vérifiée" qui conduit à agir de telle manière qu'on ait une chance optimale de trouver des données en accord avec
2. Équivalent américain de $\mathrm{l}^{\prime \prime}$ "essai francoanglais Concorde". 
ses attentes et ses croyances préalables. De nombreux travaux oublient ainsi le plus souvent de distinguer ce qui tient aux difficultés d'accès aux soins des toxicomanes dans le contexte de couverture limitée par l'assurance maladie aux États-Unis, de ce qui au contraire tient à des tendances comportementales à la non-observance intrinsèquement associées à la toxicomanie active. On sait pourtant que la perception subjective de leur état de santé et de leurs symptômes par les individus joue un grand rôle dans la détermination des comportements effectifs de demande de soins qu'ils adressent au système médical (Bishop, 1994 ; Sarafino, 1994). On connaît également la grande variabilité socioculturelle de ces perceptions : tous les individus n'interprètent pas un même symptôme de façon identique. De plus, en fonction de leur appartenance sociale, communautaire, voire de leur place dans une structure familiale, ils n'en tirent pas les mêmes conséquences en termes de décision ou non de consulter un médecin (Leventhal et al., 1983 ; Aiach et Cebe, 1991). On sait aussi que différentes formes de "détresse psychologique" peuvent affecter ces perceptions et, corrélativement, les comportements individuels d'accès aux soins (Tessler et al., 1976).

Cette intrication de déterminants multiples est de fait communément ignorée dans l'analyse des comportements d'insoumission tels que ceux qu'on attribue aux toxicomanes. On peut par exemple raisonnablement faire l'hypothèse que la consommation d'héroïne interfère négativement avec plusieurs étapes du processus qui conduit l'individu à ressentir un symptôme et à l'interpréter comme un phénomène d'ordre pathologique nécessitant un recours médical. Du fait de sa nature d'opiacé, l'héroïne interfère avec la perception des symptômes (notamment la douleur). Sa consommation peut, de plus, affecter les processus cognitifs inhérents à l'interprétation des sensations ; par exemple lorsqu'un toxicomane séropositif attribuera des douleurs d'estomac ou une diarrhée au "manque" plutôt qu'aux infections asso- ciées au VIH. La consommation d'héroïne peut également diminuer les réactions de peur face aux symptômes, en provoquant des états d'euphorie qui réduiront d'autant les motivations de recours aux soins. De façon générale, la consommation de drogue peut être utilisée comme forme d'adaptation aux conséquences pathologiques de l'infection à VIH ; elle peut aider à entretenir un déni de la nécessité du recours aux soins. Cet ensemble de conséquences psychologiques pourrait contribuer à expliquer, chez les toxicomanes infectés continuant à pratiquer l'injection, des délais plus longs entre apparition de signes cliniques et recours aux soins.

\section{Déterminations et régularités des comportements de non-observance}

Ce que semblent découvrir les professionnels de santé confrontés au VIH n'est pas nouveauté pour les observateurs des processus psychosociaux accompagnant la réalisation effective des conduites de soins (Janz et Becker, 1984; Becker, 1985 ; Ostrow et Kessler, 1993). L'insoumission discrète et le développement invisible de conduites de non-observance ont été maintes fois décrits dans diverses situations, au point que certains y ont vu le "problème le plus sérieux auquel la pratique médicale moderne a à faire face .» (Dunbar, 1990).

En matière de médicaments, une enquête française (Baudier et al., 1994) soulignait ainsi le caractère généralisé de l'auto-prescription $(59,5 \%$ de la population de plus de 18 ans déclarant prendre des médicaments « en-dehors de toute prescription médicale " et du non-respect des posologies et des durées de traitement ( $21,5 \%$ déclarant modifier les doses ou les nombres de prises journalières et $36,4 \%$ interrompre parfois des traitements avant leur terme prescrit).

De nombreuses indications suggèrent que ces pratiques décalées ne sont pas de simples effets d'étourderies ou d'ignorances mais s'appuient sur des raisonne- ments "profanes" inavoués et des postulats largement partagés. De nombreuses recherches ont mis en évidence les ajuste. ments "bricolés" par lesquels les intéressés adaptent à leurs contraintes quotidiennes et à leurs goûts les prescriptions médicales qu'ils sont supposés suivre. Ces conduites ont été observées auprès de patients atteints d'affections chroniques (diabète, insuffisance rénale, insuffisance respiratoire, etc.) (Waissman, 1990 ; Corraze, 1992), ou de patients soumis à des régimes et traitements préventifs à long terme comme pour les personnes diagnostiquées comme médicalement “à risque" parce que souffrant d'obésité ou d'hypertension (Haynes, 1976 ; Sarafino, 1994). De nombreuses études empiriques, pour l'essentiel anglo-saxonnes, se sont efforcées de traquer les déterminants supposés de ces conduites par des techniques corrélationnelles. Plus de 200 variables ont ainsi été associées à l'observance (Janz et Becker, 1984 ; Morris et Schulz, 1992). Les tendances les mieux reconnues sont les suivantes :

- la non-observance apparaît beaucoup plus souvent pour les traitements préventifs que pour les traitements curatifs ;

- l'observance est moins bonne pour le traitement des pathologies chroniques que pour le soin de pathologies en phase aiguë (Fitzgerald, 1976). Plus généralement : l'adhésion thérapeutique varie avec la durée du traitement (les thérapies longues conduisant à des taux d'observance plus faibles mais on peut avoir un déclin de l'observance même après 10 jours) (Sackett et Snow, 1979) ;

- l'observance est plus faible pour les situations caractérisées comme " asymptomatiques " que pour les situations "symptomatiques ", et diminue avec l'apparition des effets dits "secondaires" associés à la prise de médicaments ;

- la non-observance augmente avec le nombre de médicaments et la complexité de la tâche demandée au patient (d'où l'effort entrepris par tous les laboratoires pharmaceutiques pour parvenir au "dosage jour- 
nalier unique" du médicament dès que possible) ;

- un entourage qui apporte un soutien social au patient renforce généralement l'adhésion thérapeutique mais certains contextes familiaux peuvent entrainer des conséquences psychopathologiques contradictoires avec le suivi médical ;

- la forme et la qualité de la relation et de la communication médecin-malade influencent grandement le suivi d'un traitement.

En dépit d'une opinion souvent répandue dans le corps médical, ces recherches n'ont, par contre, jamais véritablement établi de corrélations significatives entre l'observance et les variables sociodémographiques classiques (âge, sexe, groupe social), du moins lorsqu'on tente d'en isoler et d'en autonomiser l'effet. La valeur prédictive de ces variables doit toujours être rapportée à leur association à d'autres facteurs contextuels comme les effets de génération ou les types de pathologie. De même, la psy. chologie différentielle s'est révélée incapable d'établir un lien entre des variations de l'observance et différentes "structures de personnalité" individuelles (Dunbar, 1990). Enfin, on ne peut que se défier, en ce domaine comme en d'autres, des prévisions stéréotypées qui chercheraient des relations de causalité directe entre observance et niveau d'instruction.

\section{Limites des modèles explicatifs}

Au-delà de ces constats empiriques, le recours à des modèles psychosociologiques à prétention explicative, comme le Health Belief Model ou "modèle des croyances sur la santé" (Becker, 1985), la théorie de l'" action raisonnée " (Terry et al., 1993), ou le modèle de "l'apprentissage social " (Bandura, 1994) a permis d'introduire des variables plus "subjectives" comme la peur face à la maladie, les motivations et les obstacles à l'action, ou les motivations générales face à la santé, ainsi que la capacité d'apprentissage social. Des relations significatives ont ainsi été mises en évidence entre des comportements d'observance d'une part et, d'autre part, l'estimation subjective des coûts matériels et psychologiques associés au traitement et à ses effets secondaires (ce que le modèle HBM qualifie de "barrières perçues " à l'action). À la différence des conduites préventives pour lesquelles on met l'accent sur la perception individuelle de la "susceptibilité" à contracter une maladie, pour l'observance de traitements c'est plutôt la dimension de "perception subjective" du bénéfice attendu de la thérapeutique qui est mise en avant comme principal facteur "explicatif" de l'observance. Malgré leur indéniable apport d'aide à la description, ces modèles n'échappent pas à un certain caractère tautologique (Hitckok et al., 1992 ; Moatti, et al., 1993) : affirmer que la " perception du bénéfice thérapeutique attendu " est un déterminant décisif de l'observance apparaît en définitive plutôt comme une mesure redondante du phénomène qu'on cherche à expliquer que comme une réelle découverte de ses mécanismes explicatifs.

\section{UN EXEMPLE D'APPROCHE PSYCHOSOCIALE : ENQUÊTES À PROPOS DE L'ESSAI DELTA}

Dans une recherche en cours (Munzenberger et al., 1996), nous nous sommes attachés à l'observation de quelques processus psychosociaux dont l'importance a pu être repérée dans le déroulement des essais cliniques. Nous avons pris pour support l'analyse d'une application locale d'un des principaux essais multicentriques internationaux en matière de sida (voir encadré).

La démarche que nous développons pour l'étude de ces essais, et qui s'efforce d'ébaucher une alternative aux modèles de type HBM, considère les phénomènes de non-observance comme des conduites et des pratiques socialement construites. Les pratiques de soin auxquelles se soumettent ou non les personnes atteintes sont ancrées sur des valeurs et des pensées collectivement 
élaborées et partagées (concernant la maladie, les traitements, la mort, le processus de guérison, le rôle du médecin). Dans cette optique, la production des comportements et leur transformation éventuelle ne peuvent se réduire à des oscillations d'attitudes ou des calculs individuels de coûts et bénéfices face à une offre médicale. Elle n'est pas non plus explicable et prévisible par le simple effet mécanique des stimulations et pressions environnementales que manipulaient les premiers psychologues béhavioristes ou comportementalistes. Les comportements des soignants et des patients prennent sens dans

\section{L'ESSAI THÉRAPEUTIQUE DELTA ${ }^{6,7}$}

Nous avons été amenés à nous intéresser plus particulièrement à l'essai Delta en raison de ses caractéristiques: longueur de l'essai (deux ans), nombre de patients important (plus de 1400 patients en France), principes actifs entraînant des effets secondaires important et posologie relativement "lourde" (cf. ci-dessous) tout ceci étant associé par ailleurs à l'existence d'un groupe placebo.

Cet ensemble de caractéristiques nous laissaient supposer que, pour cet essai, pouvaient se poser des problèmes de deux types liés à la non-observance :

- Des problèmes de types quantitatifs : le retrait de patients du protocole (notamment les "perdus de vue") entraîne une diminution de la puissance statistique et une fragilisation des résultats éventuellement obtenus.

- Des problèmes de types qualitatifs : plus graves, car moins visibles, la non-observance des prescriptions médicales ou des restrictions demandées ou "allant de soi" (problèmes des thérapies "sauvages" parallèles qu'ils s'agisse de médecines dites "douces" ou, surtout, de l'accès invisible à certains produits actifs ou antiviraux) peut brouiller la donne et fausser les résultats.

Delta est un essai international et multicentrique (Allemagne, Australie, France, GrandeBretagne, Pays-Bas et Suisse), mené sous la responsabilité de l'Agence Nationale de Recherche sur le Sida française (ANRS) et du Medical Research Council britannique (MRC). II s'agit d'un essai comparatif portant sur trois groupes et randomisé* en double aveugle pour évaluer l'efficacité et la tolérance des associations $A Z T+$ ddl et AZT + ddC, par rapport à la monothérapie de référence $A Z T$ + placebo.

\section{Critères d'inclusion dans l'essai}

Le patient doit soit être séropositif asymptomatique (groupe II et III, CDC, 1987 et ARC) et avoir un taux de lymphocytes $T 4<350 \mathrm{~mm}^{3}$, soit présenter un sida et avoir un taux de lymphocytes $\mathrm{T} 4>50 \mathrm{~mm}^{3}$.

\section{Deux sous-essais :}

- Delta 1 (Essai ANRS 017 [A]) qui s'adresse à des sujets qui n'ont jamais reçu de traitement antirétroviral. En France 1227 patients y ont été inclus.

- Delta 2 (Essai ANRS 017 [B]) qui s'adresse à des sujets qui ont déjà reçu un traitement antirétroviral pendant au moins trois mois. En France 182 patients y ont été inclus.

Principes actifs et posologie dans les trois groupes (deux groupes expérimentaux et un groupe de contrôle) de l'essai :

Premier groupe expérimental:

- AZT ou "Zidovudine" (Retrovir@) : par voie orale, $600 \mathrm{mg} / \mathrm{jour}$ en trois prises, pendant 2 ans. - ddl ou "Didanosine" (Videx@) : par voie orale, $400 \mathrm{mg} / \mathrm{jour}$ en deux prises, pendant 2 ans.

Deuxième groupe expérimental:

- AZT ou "Zidovudine" (Retrovir@) : par voie orale, $600 \mathrm{mg} /$ jour en trois prises, pendant 2 ans.

- ddC ou "Zalcitabine" (Hivid()) : par voie orale, $2,25 \mathrm{mg} / \mathrm{jour}$ en trois prises, pendant 2 ans.

Groupe contrôle :

- AZT ou "Zidovudine" (Retrovir@) : par voie orale, $600 \mathrm{mg} / \mathrm{jour}$ en trois prises, pendant 2 ans.

- Placebo de ddl ou de ddC.

Le suivi du patient

II doit être effectué pendant deux ans au moins et comporte :

- des examens cliniques tous les mois pendant les 6 premiers mois puis tous les 2 mois pendant les mois restants.

- des examens biologiques 1 mois sur 2 ou 2 mois sur 3.

6. ARCAT-SIDA, (1995). Répertoire des essais thérapeutiques en France, $3^{\circ}$ Édition, ARCAT-SIDA Eds, pp. $39-40$.

7. ANRS, (1994). Essais therrapeutiques dans l'infections par le VIH menés sous l'égide de l'Agence Nationale de Recherches sur le SIDA, ANRS Eds, fiches $n^{\circ} 017(A)$ \& 017(B). des pratiques et des actions. Ils sont "guidés", comme par une boussole, par des systèmes de représentations sociales et d'attitudes qui sont "activés" selon les contextes dans les. quels des acteurs sociaux, groupes ou individus, entrent en interaction, en relation à certains buts, sous des contraintes définies. Il s'agit ainsi d'identifier et d'expliciter la composition et la fonction de ces systèmes supposés d'articulation entre attitudes et comportements, entre représentations et pratiques (Jodelet, 1989a et 1989b ; Abric, 1994 ; Morin, 1994 ; Souville, à paraître). Cette perspective conduit à donner une grande importance aux "points de vue" profanes des personnes impliquées dans une logique d'action. Elle amène à une démarche de terrain d'écoute et d'observation. Méthodologiquement, elle nécessite un temps important d'enquête qualitative indispensable pour tenter de reconstruire les systèmes d'interprétation et les logiques d'action des personnes impliquées (voir encadré).

Dans l'exemple évoqué, soignants et patients ont successivement été interrogés. Quatre types de patients infectés par le VIH et concernés par l'essai Delta ont été identifiés et écoutés dans un des sites de recrutement de l'essai : ceux qui sont officiellement censés avoir poursuivi de manière correcte le protocole prescrit ${ }^{3}(n=15)$; ceux qui ont officiellement interrompu le protocole pour des raisons autres que les critères médicaux explicitement prévus par celui-ci $(n=7)$; ceux qui ont refusé de participer à ce même essai lors de la proposition d'inclusion $(n=6)$; ceux qui étaient éligibles sur les critères médicaux d'inclusion lors du lancement de l'essai, mais que les médecins ont écarté $a$ priori de celui-ci $(n=5)$. Une cinquième catégorie, celle des perdus de vue en cours d'essai, était bien évidemment très importante, mais se révélait par définition inaccessible ${ }^{4}$. Cette investigation qualitative conduit à considérer la non-observance comme un ensemble de comportements dont la production peut être reconstruite en ques. tionnant les pratiques de " commencement " (l'inclusion), les pratiques de maintien (le 


\section{MÉTHODOLOGIE}

Recherche rétrospective réalisée en trois étapes à Marseille dans un hôpital de jour accueillant des patients infectés par le VIH :

- Analyse de tous les dossiers de patients infectés par le VIH inclus ou éligibles dans l'essai Delta au 31 mars $1994(n=122)$ dans I'hôpital de jour.

- Enquête exploratoire par entretiens auprès de soignants $(n=9)$ : chef de service, monitrice des essais cliniques, technicienne des essais cliniques, cadre infirmier, médecins [hospitaliers, vacataires] et infirmiers de l'hôpital de jour.
- Enquête par entretiens approfondis (semidirectifs) auprès de 33 patients infectés par le VIH suivis à I'hôpital de jour. Après chaque entretien, le médecin (soit le Moniteur Essais Cliniques pour les patients en essai, soit le médecin traitant pour les patients hors essai) remplissait un questionnaire détaillé sur le patient qui venait d'être vu en entretien. Ce questionnaire recueillait des informations sur des variables de types socio-démographiques, médicales et psychosociologiques ainsi que des évaluations de l'observance des patients concernant les traitements et les rendez-vous. suivi) et les pratiques de terminaison (fin ou sortie de l'essai).

\section{Le processus d'inclusion comme raisonnement sélectif du soignant}

Pour de nombreux cliniciens, l'assurance d'une bonne observance thérapeutique passe d'abord par une estimation anticipée des facteurs qui pourraient faire obstacle au respect des prescriptions requises. En pratique, ceci conduit à limiter la participation aux essais thérapeutiques de patients (toxicomanes, membres de minorités ethniques) suspectés $a$ priori d'être peu susceptibles de se soumettre aux protocoles (Broers et al., 1993 ; Meisler et al., 1993). Notre enquête confirme la fréquence de ce processus. Elle montre que la sélection par les médecins des patients susceptibles d'être inclus dans des essais est conditionnée non seulement par les "critères officiels" d'inclusion, mais aussi par des "critères psychosociologiques" d'exclusion employés "spontanément" par les praticiens et résultant d'une culture collective "de service". Tout se passe comme si des "théories", implicites mais volontiers explicitées, de l'observance et de la non-observance guidaient les choix d'inclusion dans les essais thérapeutiques.

Ce système de représentations des capacités des patients a trois principaux points d'ancrage auxquels sont associés dans l'esprit des médecins interrogés des risques anticipés de non-observance :
- Le mode de contamination permet de particulariser (ou de stigmatiser) les pratiques toxicomaniaques. Il est perçu d'emblée comme un facteur probable de non-observance en raison des types de personnalité et de fonctionnement psychologique qui sont attribués aux toxicomanes : " Allez rendre compliants les toxicomanes ! Ces gens-là dérangent. " explique un médecin hospitalier.

- Le style de vie est pris en compte quand on lui associe la précarité, l'instabilité et la désinsertion sociale. Les déclarations recueillies auprès de soignants illustrent bien ce raisonnement sélectif qui tente d'évaluer la fiabilité mais aussi la disponibilité des patients. L'observance thérapeutique présuppose un mode de vie stable : ceux qui changent sans arrêt de mode de vie sont des mauvais candidats. Ce ne sont pas forcément des toxicomanes. " Il y a des critères officiels et ensuite, il y a le profil psychologique du patient, son mode de vie qui nous font penser que le patient pourra adhérer. Est-ce qu'il a des enfants à la maison? Estce qu'il se drogue ou pas? A-t-il un métier ou pas? A-t-il envie de prendre un traitement ou pas...? " . "J'aurai moins tendance à faire rentrer une femme qui a des enfants, par rapport à sa disponibilité ".

Mais paradoxalement l'image d'un mode de vie stable et régulier peut devenir une contre-indication si la stabilité est associée à des contraintes d'emploi du temps
3. Il est à noter que nous avons inclus dans ceux-ci une patiente qui a rigoureusement suivi le protocole jusqu'à ce qu'il soit interrompu pour raison médicale.

4. Sauf à se livrer à des recherches qui auraient nécessité une remise en cause de l'anonymat sans accord préalable du patient et qui auraient été contraires aux règles éthiques des essais cliniques. 
rigides. L'emploi du temps non modifiable des fonctionnaires peut ainsi devenir, pour l'un des médecins interrogés, un argument de contre-indication : "Pour les fonctionnaires, ça pose de gros problèmes, car ils ne peuvent pas adapter leurs horaires pour les consultations ".

- Enfin, l'appréciation subjective par le médecin du niveau intellectuel du patient particularise l'incapacité à comprendre et respecter les règles d'un protocole, souvent sur la base des difficultés rencontrées antérieurement dans la relation médecin-malade. "Certains ont un quotient intellectuel... Ils ne comprennent pas. Vous leur expliquez pendant un quart d'heure quand venir la prochaine fois, comment prendre le médicament... Ils n'arrivent pas à comprendre. Alors dans un essai... "

\section{L'inclusion comme un acte d'acceptation qui différencie les patients}

Pour les patients, l'acceptation de l'essai est plus ou moins volontairement assumée et pas toujours associée à un sentiment d'engagement. D'une manière générale, la manière dont l'essai sera accepté et adopté dépend de l'état clinique tel que l'évalue subjectivement le patient au moment de la proposition d'inclusion. Cette différence initiale joue un rôle important dans la variabilité future de l'observance. Un état "asymptomatique" ne conduit pas à la même représentation de l'essai qu'un état symptomatique.

- Les patients symptomatiques, qui présentent déjà, avec plus ou moins d'intensité, des signes cliniques associés au Sida, vont plutôt envisager l'essai comme une "lutte du dernier recours ", une solution pour " ralentir le processus ", un « espoir de mieux vivre " : « ... de toute façon, plutôt que de rien faire, moi, je préfère essayer : [...] je suis partant et puis, au point où j'en suis, j'ai plus rien à perdre. " (homme, 49 ans, contaminé par voie hétérosexuelle, "observant” de l'essai Delta). « ... Je peux avoir une espérance de vie plus longue et peut-être une espérance de vie normale "(femme, 31 ans, contaminée par voie toxicomane, "observante" de l'essai Delta).

- Les patients "asymptomatiques" semblent fonder leur acceptation sur deux raisonnements principaux : un raisonnement mettant au premier plan l'importance qu'ils accordent à un suivi personnalisé ; ils ont alors le sentiment que l'équipe hospitalière leur est reconnaissante de leur participation à l'essai. Un deuxième type de raisonnement insiste sur l'importance des découvertes médicales et l'intérêt à en être les premiers bénéficiaires tout en contribuant à aider les autres personnes atteintes. L'argument le plus saillant et le plus valorisé semble cependant bien être l'attente d'un meilleur suivi. Malgré les informations détaillées fournies par l'équipe médicale lors de l'inclusion, le concept d'essai est généralement flou et très mal différencié de la notion de traitement. On lui associe surtout des contraintes qui sont comme le prix à payer pour un meilleur suivi.

A contrario, l'analyse des raisons de refus des patients asymptomatiques lors des sollicitations d'entrer dans un protocole comme Delta révèle l'emprise de représentations pouvant faire obstacle à une volonté active de participer à un essai thérapeutique. Les mobiles de refus, qui peuvent pour d'autres devenir des causes ultérieures de non-observance en cas d'entrée réticente en protocole, mettent au premier plan les inconvénients associés à la notion d'essai : contraintes temporelles imposées et vécues comme une perturbation d'un mode de vie (en particulier par rapport aux horaires de travail) ; rejet de l'adoption d'un rôle de malade alors que le patient veut continuer à se représenter la séropositivité comme un état asymptomatique, socialement invisible et radicalement différent d'un état de maladie : «... je voulais pas faire ma vie autour de la séropositivité et là c'était carrément faire ma vie autour de ça " (homme, 37 ans, contaminé par voie homosexuelle, a refusé de participer à l'essai Delta) ; pression mal ressentie pour se soumettre à une équipe de recherche et crainte d'abandonner (ou d'être abandonné par) le médecin qui, jusque là, a assuré le suivi, au bénéfice d'un spécialiste du suivi des essais cliniques.

- C'est parmi ceux qui refusent qu'on retrouve le plus fréquemment les mots "cobaye" et "placebo" ainsi que la revendication d'un droit de savoir qu'exclut la méthodologie des essais : " Les protocoles je les ai refusés. Parce que je n'avais pas envie de le faire... On vous le dit au bout de quatre mois si c'est un placebo... moi les médicaments que je prends c'est pas des placebos " (femme, 37 ans, contaminée par voie toxicomane, a refusé de participer à l'essai Delta). Ces patients se positionnent contre une pratique qui, à leurs yeux, anonymise et dépersonnalise le malade. Ils refusent de se soumettre aveuglément aux propositions des médecins.

Une autre argumentation, répandue parmi ceux qui refusent, associe l'entrée dans un essai à une entrée en maladie. Ce raisonnement est d'autant plus sensible que le sujet avant l'essai n'a jamais eu recours à un traitement antiviral contre le sida. En témoignent rétrospectivement des patients qui n'ont accepté d'entrer dans un essai qu'après une phase de refus explicitement associée à la peur d'un passage inexorable vers la maladie : " Le jour où il a fallu que je passe vraiment sous un traitement j'étais catastrophé " (homme, 39 ans, contaminé par voie homosexuelle, a interrompu le protocole de l'essai Delta). « Il y a eu un passage difficile entre le moment où on se sait séropositif et le moment où il faut prendre le traitement " (homme, 37 ans, contaminé par voie homosexuelle, a refusé de participer à l'essai Delta).

\section{Le suivi d'un essai comme pratique d'observance conditionnelle et contextualisée}

Quelques propositions permettent de caractériser les principaux résultats des observations recueillies auprès des patients engagés dans les protocoles étudiés.

On ne rencontre qu'exceptionnellement des patients qui suivent à la lettre les 
prescriptions médicales pendant toute la durée du traitement telle qu'elle est délimitée par les contraintes d'un protocole. Les formes repérables de non-observance sont variées, mais il est commode de distinguer les écarts d'observance présentés comme "involontaires" (oublis occasionnels de prise de médicaments) et les écarts ou transgressions délibérés ou "volontaires" (prises groupées de médicament, arrêt de traitement pour des choix liés au style de vie comme les arrêts à l'occasion des "vacances").

On peut être non-observant "en toute innocence", sans même songer à en aviser un médecin qui percevra une bonne volonté manifeste confirmée, par exemple, par le respect des rendez-vous. Certains patients ainsi sont jugés "observants" par les médecins, mais en fait adaptent de manière autonome leur traitement à leur mode et rythme de vie. Ils distinguent l'objectif "prendre la quantité $\mathrm{x}$ de médicaments" de l'objectif "prendre toutes les $\mathrm{x}$ heures $\mathrm{x}$ médicaments". Dans cette stratégie, l'important pour eux est de respecter le contrat du nombre de médicaments à prendre par jour plutôt que la fréquence des prises de médicaments. Ainsi certains patients prendront le traitement en bloc, en une seule prise, tous les soirs. Ce comportement d'adaptation libre des prescriptions peut encore être davantage accentué pour les patients en traitement ne participant pas à un essai : « J'avais des infections, des angines, beaucoup de rhinos, des grippes, des conneries. On m'en a prescrit un par jour. Là j'en prends un, deux par jour. Quand je sens que je suis un peu fébrile, j'en prends un le matin et un le soir " (homme, 46 ans, contaminé par voie homosexuelle, a refusé de participer à l'essai Delta). “ C'est déjà bien pour moi, d'arriver à les prendre ! " (femme, 37 ans, contaminée par voie toxicomane, a refusé de participer à l'essai Delta).

On peut être un non-observant dissimulé qui ne parle pas au médecin des écarts au protocole qu'il s'autorise. Tel ce patient n'osant pas dire au spécialiste hospitalier qu'il voyait en parallèle un médecin homéopathe : « ...au début je n'osais pas lui dire que je voyais cette personne en même temps parce que j'avais peur qu'il pense que je n'ai pas confiance " (homme, 44 ans, contaminé par voie homosexuelle, a refusé de participer à l'essai Delta).

Surtout, chaque patient ne s'installe pas de manière définitive dans l'observance ou la non-observance entre l'entrée et la sortie d'un essai. Des événements et des moments critiques marquent l'histoire des patients dans leur relation à l'essai. Certains de ces événements sont inducteurs de fidélité aux prescriptions : l'aggravation d'un état de santé ou au contraire une amélioration de l'état attribuée, à tort ou à raison, au traitement. D'autres événements au contraire poussent à la rupture ou à la transgression, avouée ou non, de ce qui est prévu par l'organisation temporelle du protocole : l'information médiatique mettant en avant l'inefficacité ou les risques d'un médicament ${ }^{5}$ ou la rencontre d'un autre patient en essai, dont l'état de santé s'est brusquement aggravé.

Les peurs, émotions et inquiétudes, comme aussi les demandes et fantasmes associés aux mystères des protocoles risquent de se rigidifier dans des raisonnements défensifs fondés sur les contradictions ressenties entre santé et prise de médicaments. Dans une argumentation de cohérence inquiète, on refuse très logiquement le médicament qui peut rendre malade alors qu'on ne l'est pas encore. Usant du même type de raisonnement articulé sur d'autres images dont le médicament est porteur, des patients symptomatiques deviendront volontiers demandeurs et consommateurs de traitements ou de produits "parallèles" référant à une médecine "naturelle" et non "médicale" et ne relevant donc pas d'un étiquetage "médicamenteux" : " ils ont un très bon produit. C'est de la nourriture, c'est pas un traitement. Elle m'a dit : "écoutez, monsieur, si ça marche pas je vous rembourse".. donc c'est quand même... c'est des produits de Chine fabriqués par les Américains... alors je vais essayer et de toute façon, ça pourra pas me faire de mal... De toute façon, c'est de l'alimentation, c'est pas un médicament " (homme, 49 ans, conta-
5. Ce qui s'est passé après la publication des résultats de l'essai Concorde en 1993 qui ne montrait pas d'impact significatif sur la survie de la prise d'AZT au stade asymptomatique. 
miné par voie hétérosexuelle, "observant" de l'essai Delta).

Un critère toutefois est très largement utilisé pour évaluer le rôle et l'efficacité d'un médicament de traitement de l'infection par le VIH : les T4 (ou CD4+). La plupart des patients apprennent que lorsque les T4 "descendent", le moment de suivre un traitement ou d'entrer dans un protocole est arrivé. La référence aux $\mathrm{T} 4$ peut ainsi devenir une arme à double tranchant dans l'effort des médecins de persuader les patients de l'intérêt d'entrer dans un protocole. On s'appuie sur leur chute pour renforcer l'acceptation de l'idée d'essai : «Oui, on me l'a proposé quand les T4 sont descendus en dessous de 280 je crois... Donc là on m'a proposé un protocole " (homme, 31 ans, mode de contamination inconnu, " observant " de l'essai Delta). " Quand je suis arrivé là, mes $\mathrm{T} 4$ avaient diminué, et elle a voulu absolument que je prenne l'AZT " (homme, 37 ans, contaminé par voie homosexuelle, "observant" de l'essai Delta). Mais si à l'inclusion dans l'essai n'est pas associée une remontée durable de $\mathrm{T} 4$, on peut produire un découragement ou un abandon ou une diminution d'observance. "Je me retrouve avec un 15 en T4, c'est pas beaucoup " (homme, 38 ans, contaminé par voie homosexuelle, “observant” de l'essai Delta).

Enfin, les conduites d'observance se construisent dans des espaces relationnels, marqués par un clivage entre des espaces de paroles et de confiance et des espaces de défiance, de calcul et de dissimulation. Nous avons retrouvé à propos de l'essai Delta ce qu'on trouve en d'autres études d'observance : l'importance capitale de la relation au médecin-interlocuteur. Les patients qui acceptent l'essai font état majoritairement de leur confiance en leur médecin. Mais ce rapport de confiance est accompagné de deux types différents de soumission à son influence. Certains s'en remettent à lui presque aveuglément. D'autres ont une démarche plus active de recherche d'informations, qui s'appuie sur des valeurs et des engagements conduisant à une théorie des essais explicitement plus élaborée que pour les premiers. Ces patients dits "actifs" peuvent se conduire comme des chercheurs amateurs. Ils sont plus "observants" de manière persistante malgré les gênes ou inquiétudes rencontrées à l'occasion d'apparition d'effets secondaires dus au traitement. Ils sont en revanche plus exigeants et critiques et donnent une grande importance au soutien médical et/ou psychologique.

Nous avons pu aussi noter, dans les itinéraires d'entrée en protocole et de maintien dans l'essai, l'importance des tiers qui s'interposent entre le médecin et le patient en essai :

- le tiers qui assure le soutien informationnel ou clarificateur, qui valide ou infirme la légitimité de la prescription thérapeutique, cette place pouvant être tenue par un médecin généraliste : " Il m'explique très bien les choses, à ma portée, à mon niveau donc ça m'aide, oui " (femme, 31 ans, contaminée par voie toxicomane, "observante" de l'essai Delta). Ce peut être aussi un autre professionnel de santé ou une association;

- le tiers qui apporte le soutien émotionnel ou instrumental : " Je peux pas les oublier les médicaments ! surtout qu'il y a mes parents à côté pour me le rappeler. " (homme, 31 ans, mode de contamination inconnu, "observant" de l'essai Delta). « Les personnes qui sont dans ma famille acceptent déjà difficilement certains aspects de ma personnalité,... elles sont pas en mesure d'accepter... cela ne ferait que les encombrer " (homme, 36 ans, contaminé par voie homosexuelle, a interrompu le protocole de l'essai Delta).

\section{Sciences sociales et recherche clinique : la nécessaire confrontation impossible}

On ne peut que constater la rareté des travaux de recherche consacrés à l'étude de ces problèmes dans le système de soins français. Le développement d'une psychologie sociale de la santé en France reste embryonnaire malgré le mouvement d'intérêt suscité par la visibilité de l'épidémie de sida. Le déficit et le décalage par rapport au leadership nord-américain mais aussi par rapport à d'autres pays européens sur ce terrain, persiste tout autant dans la collecte de données empiriques contrôlées que dans l'élaboration de modèles théoriques porteurs de renouvellement heuristique.

Les premières investigations empiriques que nous avons menées sur le terrain délimité de la mise en œuvre des essais thérapeutiques rejoignent les observations d'autres chercheurs qui se sont consacrés à l'écoute et à l'étude des conduites de soin et des problèmes que rencontrent les personnes atteintes par le sida. Ainsi des travaux de recherche sur un thème sensible comme celui de la transfusion sanguine (Setbon, 1993), l'analyse des réactions du personnel infirmier à la prise en charge des patients infectés par le VIH (Giami et Veil, 1995) ou encore la prise en charge des toxicomanes dans les services hospitaliers (Lert et Marne, 1992) ont pu être réalisés. Une conclusion en forme d'hypothèse provisoire peut être dégagée de ces rares questionnements de terrain qui ont eu pour caractéristique commune de recourir à des investigations qualitatives approfondies avant d'envisager de mettre en place des dispositifs de validation quantitatives : on peut considérer que risquent de se développer aujourd'hui de nouvelles formes de "divorce" entre le corps médical et les communautés de malades. Ce phénomène relève d'un questionnement pluridisciplinaire qui arrache l'analyse aux polémiques circonstancielles et aux reconstitutions tautologiques pour mieux caractériser les obstacles que rencontre maintenant l'innovation dans la recherche médicale qui intervient sur des pathologies socialement "sensibles" comme l'infection par le VIH. Plusieurs pistes d'analyse et d'intervention nous paraissent mériter une attention particulière.

En premier lieu, on a l'effet d'un processus socio-cognitif très général. Pour le sida comme pour d'autres pathologies, des différences sans doute irréductibles continuent et continueront à opposer des représentations "profanes" de la maladie et du soin 
et des représentations expertes ou professionnelles (Herzlich et Pierret, 1984 ; Herzlich, 1986 ; Laplantine, 1986 ; Flick, 1993). Ces distinctions structurelles et communicationnelles entraînent inévitablement des conflits virtuels d'interprétation et des probabilités d'insoumission à la logique professionnelle médicale. Les sciences sociales appliquées depuis quelques années dédramatisent la persistance de ces écarts. Elles tendent à affirmer en direction des professionnels que les différences de perspectives ne deviennent dysfonctionnelles que si on les dénie et qu'en revanche la prise en compte compréhensive, "négociée" ou discutée de ce que les professionnels appréhendent, de façon unilatérale, comme des "fausses croyances" peut en réalité aider à produire des comportements de prévention et de soin mieux adaptés.

En second lieu, une question déstabilise profondément l'équilibre fragile qui commençait à s'établir entre les représentations scientifiques de la pathologie et les représentations sociales circulant parmi les patients et le grand public autour d'une distinction entre séropositivité et maladie : faut-il intervenir précocement ou au contraire attendre des signes incontestables de passage à la maladie (Fontenay, 1995) ? Le nouveau consensus scientifique, qui tend à se constituer actuellement en faveur d'une intervention thérapeutique de plus en plus précoce, si possible dès la phase de primoinfection, est potentiellement porteur de difficultés majeures. Il est indéniable que la distinction entre séropositivité et maladie est un trait caractéristique et qui était en voie de stabilisation dans l'émergence d'une représentation du sida dans l'espace public (Calvez et al., 1994). Comme l'a montré la première recherche sociologique approfondie sur la vie quotidienne des personnes infectées en France (Carricaburu et Pierret, 1995), cette distinction guide implicitement ou explicitement la gestion individuelle de l'infection par la majorité des patients. La séropositivité, ces dernières années, pouvait ainsi être perçue comme un état de transition. Dans cet état de transition, un sens social pouvait et devait être attribué sans que domine à ce stade l'emprise d'un codage et d'un encadrement médical lourd.

On peut donc s'attendre à ce qu'un bouleversement du message médical conduisant à réintégrer, pour les légitimes besoins de la prise en charge, la totalité de l'histoire naturelle de l'infection dans la catégorie de la "maladie" ne provoque une "crise" au moins temporaire, des relations médecin/malade. Si cette hypothèse s'avère exacte, elle attire l'attention sur la nécessité de combler rapidement le déficit, à notre connaissance total en France, de recherches évaluatives de différentes formes d'intervention psychologiques, psychothérapeutiques et d'accompagnement social visant à mieux soutenir les patients, et dans certains cas à renforcer l'observance (Chesney, 1993). Il devrait, en tout cas être recommandé que tout nouvel essai clinique soit accompagné de modalités précises de suivi psychologique, cette condition créant alors la possibilité de recherches évaluatives et comparatives sur différentes modalités d'intervention.

C'est à cette articulation de la recherche et de l'action sur un terrain en crise et en proie au changement que travaille notre projet actuel.

\section{Références}

Abric J.C. (1994). Les representations sociales : aspects théoriques, in Pratiques sociales et représentations, J. C. Abric (Eds.), Paris, PUF.

Aiach P. et Cebe D. (1991). Expression des symptômes et conduites de maladie, Paris, INSERM/Doin.

Bandura A. (1994). Social cognitive theory and exercice of control over HIV infection, in Theories and methods of beha. vioral interventions, Diclemente R.J. \& Peterson J.L.(Ed.), New York, Plenum Press.

Baudier F., Dressen C. et Alias F. (1994). Baromètre SANTÉ 92 Enquète annuelle sur la Santè des Français, Paris, Comité Francais d'Education pour la Santé.

Becker R et al. (1993). Unblinding a double-blind Zidovudine (ZDV) clinical trial by analysis of MCV values in IXth International Conference on AIDS, Berlin, Abstract book, 1(POB44-2547), 559.

Becker M.H. (1985). Patient adherence to prescribed therapies Medical Care, 23, 539-555.

Bishop G.D. (1994). Understanding the understanding of illness : Lay disease representations, in Mental representations in health and illness, J.A. Skelton \& R.T. Croyle (Eds.), New York, Springer-Verlag, 32-59.

Bouvenot G. \& Vray M. (1994). Essais Cliniques, théorie, pratique et critique, Paris, Flammarion coll. Médecine-Sciences. 
Broers B., Morabia A., and Hirschel B. (1993). Factors determining compliance of intravenous drugusers with Zidovudine treatment. Paper presented at the $I X$ International Conference on AIDS, (pp. 821 (abstract PO-D08-36-21), Berlin.

Calvez M., Paicheler G. et Souteyrand Y. (1994). Connaissances, représentations, comportements : sciences sociales et prẻvention du Sida, Paris, ANRS.

Carricaburu D. et Pierret J. (1995). From biographical disruption to biographical reinforcement : the case of HIV-positive men, Soc Hith \& III, 17, 65-88.

Chambon J.F. (1995). Les implications des essais ACTG 175 et Delta : Paradoxe, Le Journal du Sida, (78), 4-8.

Chesney M.A. and Folkman S.A. (1994). Psychological impact of HIV disease and implications for intervention, Psych Clin North America, (17), 163-181.

Chesney M.A. (1993). Health Psychology in the 21st century : Aids as a harbinger of things to come, Heath Psychol, (12), 259268.

Corraze J. (1992). Psychologie et Médecine, Paris, PUF. Dormont J. (1992). Les essais à l'épreuve, Autrement, (130), 67 78.

Dunbar J. (1990). Predictors of patient adherence : patient characteristics, in The handbook of health behavior change Shumaker, S.A. et al. (Eds.), New-York, Springer Pub Co.

Fauché N., Obadia M., Bicart-See A., Partisani M., Lapeyre M. Beslay C., and Auvergnat J. C. (1994). Participation of HIV positive patients in clinical trials : motivations, psychological sup port and effects, Paper presented at the Xth International Conference on Aids, Yokohama, Japan.

Fitzgerald J.D. (1976). The influence of the medication on compliance with therapeutic regimens, in Compliance with the rapeutic regimens, D.L. Sackett and R.B. Haynes (Eds.) Baltimore, John Hopkins University Press.

Flick U. (1993). La perception Quotidienne de la santé et de la maladie, Paris, L'Harmattan.

Fontenay F. (1995). Traiter dès la primo-infection ?, Le Journa du Sida, 78, 9-11.

Garnier M., Delamare V., Delamare J. et Delamare-Riche T. 1992). Dictionnaire des termes de médecine, $23^{\circ}$ édition, Paris. Giami A. et Veil C. (1995). Des Infirmières face au sida, Paris, Éditions INSERM.

Grmek M.D. (1989). Histoire du Sida, Paris, Payot.

Haynes R.B. (1976). A critical review of the "determinants" of patient compliance with therapeutic regimens, in Compliance with therapeutic regimens, D.L. Sackett and R.B. Haynes, Baltimore, The John Hopkins Univ. Press.

Herzlich C. (1986). Représentations sociales de la santé et de la maladie et leur dynamique dans le champ social, in L'étude des représentations sociales, W. Doise et A. Palmonari (Eds) Neuchatel, Delachaux-Niestlé.

Herzlich C. et Pierret J. (1984). Maladies d'hier, malades d'aujourd'hui. De la mort collective au devoir de guérison, Paris, Payot.

Herzlich C. et Pierret J. (1988). Une maladie dans l'espace public : le Sida dans six quotidiens français, Annales ESC, 5 , 1109-1134.

Hitckock P.B., Obantley P.J., Jones G.N. and Mc Knignt G.T (1992). Stress and social support as predictors of dietary compliance in hemodialysis patients, Beh Med, 18, 13-20.

Ho D., Neumann A.U., Perelson A.S., Chen W., Leonard J.M. and Markowitz M. (1995). Rapid turnover of plasma virions and CD4 lymphocytes in HIV-1 infection. Nature, 373, 123-126.

Janz N.K. and Becker M.H. (1984). The Health Belief Model : a decade later. Health Education Quarterly, 11, 1-47.

Jodelet D. (1989a). Folies et Représentations Sociales, Paris, PUF. Jodelet D. (1989b). Représentations sociales : un domaine en expansion, in Les Représentations sociales, D. Jodelet (Eds), Paris, PUF.

Joule R.V. et Peyrolle F. (à paraitre). Engagement et prévention contre le sida, in El desafio del SIDA, N. Basabe, D. Paez, R. Deschamps et H. Paicheler (Eds), Madrid, Fundamentos. Katz m., bindman a. and Komaromy M. (1992). Coping with Hiv infection : why people delay care. Ann Intern Med, 117, 797. Laplantine F. (1986). Anthropologie de la maladie, Paris, Payot. Lazar P. (1996). L'éthique biomédicale en question, Paris, Liana Levi.

Lert F. and Marne M.J. (1992). Hospital care for drug users with AIDS or HIV infection in France, AIDS Care, 4, 3, 333-338.

Leventhal H., Safer, m.a. and Panagis D.M. (1983). The impact of communications on the self-regulation of health beliefs, decisions, and behavior, Heatlh Educ Quart 10: 3-29.

Meisler A., Ickovics J., Skowronski C., Walesky M., Fielin M. and Friedland G. (1993). Adherence to clinical trials among women minorities and IV drug users, Paper presented at the IXth International Conference on AIDS, Berlin, June 6-11.

Moatti J.P., Dab W. et Pollak M. (1992). Les Français et le sida, La Recherche, 247, 1202-1211.

Moatti J.P., Beltzer N. et Dab W. (1993). Les modèles d'analyse des comportements à risque face à l'infection à VIH, Population, 5, 1505-1534.

Morin M. (1994). Entre représentations et pratiques : le sida, la prévention et les jeunes, In Représentations et Pratiques Sociales, J.C. Abric (Eds), Paris, PUF

Morin M. et Joule R.V. (1991). Représentations sociales et engagement. Recherches psychosociales en milieu scolaire sur les modifications des conduites des jeunes en relation au sida. Aix-en-Provence, INSERM-ANRS, Laboratoire de psychologie Sociale - Université de Provence.

Morris L.S. and Schulz R.M. (1992). Patient compliance - an overview, J. of Cinical Pharmacy and Therapeutics, 17, 283-29. Munzenberger N., Souville M., Morin M. et Moatti J.P. (1996). Approche psycho-sociale de l'observance therapeutique et de '"adherence" aux essais cliniques chez les patients infectés par le VIH, Paris, ANRS.

\section{ABSTRACT : Compliance in Clinical Trials among HIV-Infected Patients : the Contribution of Psychosocial Research}

Compliance in AIDS clinical trials is a critical issue for progress in medical research and improvement for care and cure of HIV and Aids patients. This paper presents data showing that noncompliance is a widespread and neglected social process. It can be observed in behaviors which ignore or forget medical recommendations in primary prevention as well as in clinical trials whose scientific results can be endangered by dropouts or non-recognized transgressions of medical prescriptions. Social-psychological models like the Health Belief Model did not succeed so far to explain and predict non-compliant behaviors in a satisfactory way. Previous empirical research data, collected in relation to other pathologies, could lead to a better understanding of difficulties which may not be specific to Aids medical research. A social representations approach may contribute to evaluate to what extent clinical research investigators and patients can share and nego-
Ostrow D.G. and Kessler R.C. (1993). Methodological issues in AIDS behavioral research, New York, Plenum.

Paicheler G. et Quemin A. (1994). Intégration des connaisances et logiques préventives face au risque de contamination par le VIH, in Connaissances, Représentations, comportements. sciences sociales et prévention du sida, M. Calvez, G. Paicheler et Y. Souteyrand (Eds), Paris, Eds ANRS.

Peto D., Remy J., Van Campenhoudt L. et M. Hubert M. (1992). Sida. L'amour face à la peur, Paris, I'Harmattan.

Sackett DL. and Snow J.C. (1979). The magnitude of compliance and noncompliance, in Compliance in Health Care, R. B. Haynes \& D.L. Sackett (Eds.), Baltimore, The John Hopkins University Press.

Sarafino E.P. (1994). Health Psychology, New York, Wiley. Setbon M. (1993). Pouvoirs contre sida, Paris, Seuil.

Souville M., (à paraitre). Systèmes représentationne/s des patients infectés par le VIH et conduites d'observance théra peutique, Paris, ANRS.

Stein M.D., Piette J., Mor V. et al. (1991). Differences in access to zidovudine (AZT) among symptomatic HIV-infected persons. J Gen Intern Med, 6, 35-40.

Terry D.J., Gallois G. and McCamish (1993). The Theory of Resoned Action: Its application to AIDS preventive Behavior, New York, Pergamon Press.

Tessler R., Mechanic d. and Dimond M. (1976). The effect of psychological distress on physician utilization : A prospective study, J Hith Soc Behv, 17, 353-364.

Waissman R. (1990). An analysis of doctor-parent interactions in the case of paediatyric renal failure : the choice of home dialysis, Sociology of Health and IIIness, 12 (4), $432-451$.

Yéni P. (1995). Preliminary results of the European/Australian Delta trial, Vth European Conference on Clinical Aspects and Treatment of HIV infection, Copenhague.

Yéni P. (1996). Nouvelles molécules + nouveaux marqueurs = nouvelles questions - nouveaux essais, Le Journal du Sida, (83), 21.

ciate what is at stake for them in clinical trials. In line with this framework a qualitative survey has questioned physicians and patients in an Aids medical center in the south of France in charge with therapeutic trials. It shows that implicit selection rules in enrollment practices are used by caregivers. Symptomatic and asymptomatic patients differ in the way they accept or refuse enrollment in a trial and comply to medical prescriptions. Their compliance behavior varies according to the way they evaluate their medical status and appreciate or trust physical-patient relationship. It is hypothesized that changes which have already occured in scientific knowledge of HIV treatment will increase discrepancies between physicians' representations and folk or patients' expectations. Social sciences should pay more attention to this possibility and contribute to help research investigators and health professionals to cope with incoming critical changes. 